\title{
Thoracic primary central nervous system melanoma and lumbar schwannoma of complex neurocristopathy: case report
}

\author{
Aaron Chance, BS, ${ }^{1,2}$ Jesse J. Liu, MD, ${ }^{2}$ Jeffrey S. Raskin, MD, ${ }^{2}$ Viktor Zherebitskiy, MD, ${ }^{3}$ \\ Sakir H. Gultekin, MD, ${ }^{3}$ and Ahmed M. Raslan, MD²
}

${ }^{1}$ University of Texas Medical School, Houston, Texas; and Departments of ${ }^{2}$ Neurological Surgery and ${ }^{3}$ Pathology, Oregon Health \& Science University, Portland, Oregon

\begin{abstract}
Complex neurocristopathy, a disorder resulting from the aberrant proliferation of tissues derived from neural crest cells, has been previously reported in 2 patients, both involving ophthalmic melanoma and other tumors. One patient had a periorbital neurofibroma, sphenoid wing meningioma, and choroid juxtapapillary meningioma. The other patient had a choroidal melanoma and an optic nerve sheath meningioma. The authors describe clinical and pathological findings in a patient who underwent resection of 2 distinct lesions: primary CNS melanoma at T-12 and an L-5 schwannoma. Clinical and histopathological findings of the case are reviewed. To the authors' knowledge, this is the first patient to present with complex neurocristopathy involving both a spinal melanoma and schwannoma.
\end{abstract}

http://thejns.org/doi/abs/10.3171/2015.3.SPINE141265

KEY WORDS complex neurocristopathy; spinal melanoma; melanocytoma; schwannoma; oncology

$\mathrm{N}$ EUROECTODERMAL-DERIVED neural crest cells give rise to peripheral and autonomic nervous systems, melanocytes, leptomeninges, and the chromaffin cells of the adrenal medulla, among numerous other vital structures. The term neurocristopathy has been coined to delineate a variety of disorders resulting from specific errors in the development of neural crest progeny, and is further divided into the classifications simple and complex. ${ }^{2}$ A simple neurocristopathy is characterized by a single localized aberration such as a solitary neuroblastoma, carcinoid tumor, pheochromocytoma, peripheral neuroectodermal tumor, or thyroid medullary carcinoma. Alternatively, a complex neurocristopathy is denoted by a varied assortment of simple neurocristopathies in a single patient. Represented within the complex category most frequently are the syndromes of neurofibromatosis Types 1 and 2 , with their differing diagnostic criteria including combinations of gliomas, meningiomas, malignant melanomas, neurofibromas, pheochromocytomas, and schwannomas. Many other potential complex neurocristopathies do not correspond to named syndromes and may not garner substantial clinical suspicion. However, clinicians should pay careful attention to the possibility of additional benign and malignant neural crest-derived tumors in this population as well. We report a novel complex neurocristopathy in the form of a spinal melanoma in association with a schwannoma, and differentiate this case from 2 prior reports of complex neurocristopathies in the form of ophthalmic melanomas, rather than spinal, in association with other neural crest-derived tumors.

\section{Case Report}

Presentation and Examination

A 46-year-old man initially presented to a referring facility with low-back pain and radiculopathy, at which time neuroimaging revealed 2 thoracolumbar lesions. The patient delayed treatment for 1 year when he subsequently presented to Oregon Health \& Science University neurological surgery with 1 month of worsening right lowerextremity numbness, left lower-extremity paresthesias, perineal burning sensation, and several weeks of urinary retention, requiring bladder catheterization. His medical history was significant for hypercholesterolemia, and he reported smoking cessation in 2009 with a cumulative 20 pack-year history. The patient's personal and family histo- 
ries were negative for neurofibromatosis, but positive for a sister with lung cancer and a cousin with colon cancer. Magnetic resonance imaging demonstrated a large intradural extramedullary hemorrhagic lesion at T-12, causing compression of the conus medullaris and a smaller intradural mass at L-5 (Fig. 1).

\section{First Operation}

The patient underwent a T11-L1 laminectomy with a subsequent uncomplicated perioperative course. Intraoperatively, the lesion was noted to be hemorrhagic and highly vascularized, requiring vascular clips to control large perimedullary feeding arterioles. The mass emanated from the dura, which was superficially dissected from the pia of the conus using sharp dissection. The mass was resected from the right T-12 nerve root en bloc with the dura, which was primarily repaired using Prolene and TISSEEL. An intraoperative pathological frozen section was reported as an arteriovenous malformation; therefore, no postoperative MR images were obtained. To evaluate for potential arteriovenous malformation, a spinal angiogram was obtained, which demonstrated no evidence of residual vascular lesion. However, final surgical pathology revealed primary CNS melanoma (Fig. 2).
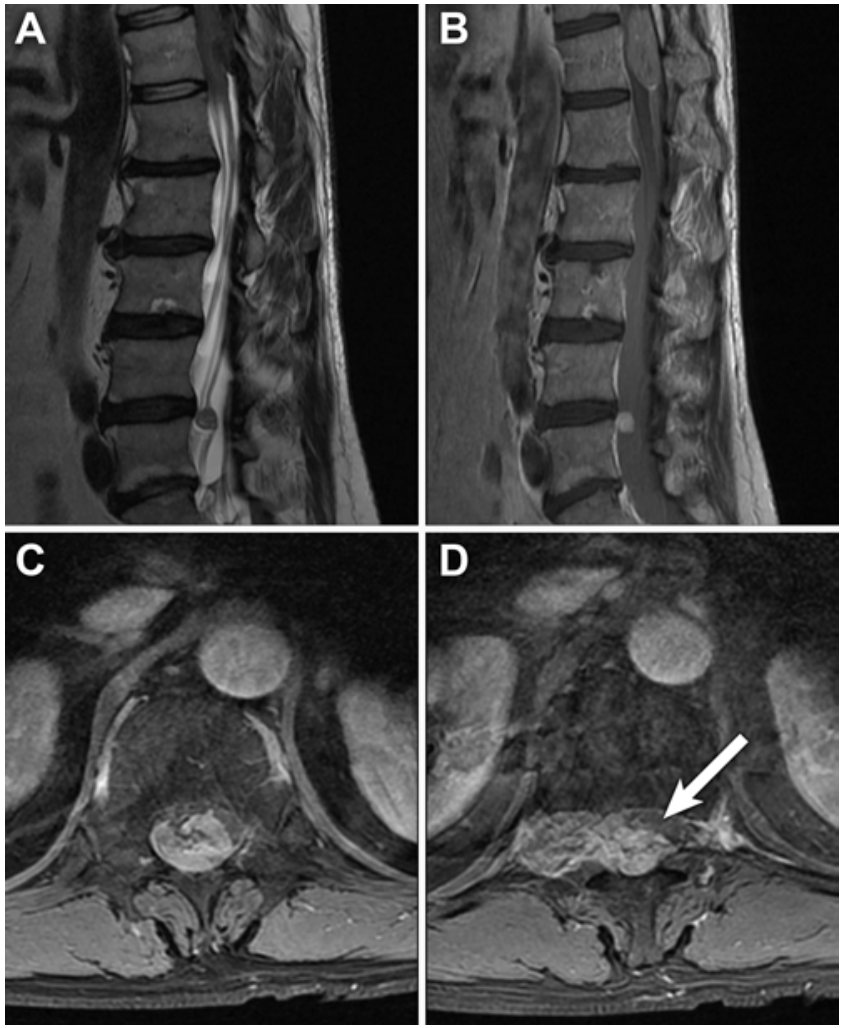

FIG. 1. Orthogonal images from presenting enhanced lumbar MR images showing tandem intraspinal lesions. A: Parasagittal T2-weighted image showing obliteration of T2 signal at the level of the conus and a small round lesion at the L-5 nerve root. B: T1-weighted postgadolinium image demonstrating enhancement within the T-12 and L-5 lesions. C and D: T1-weighted postgadolinium axial rostral (C) and caudal (D) through the T-12 lesion measuring $4.0 \times 3.9 \times 1.8 \mathrm{~cm}$, showing compression of and displacement of the conus leftward.

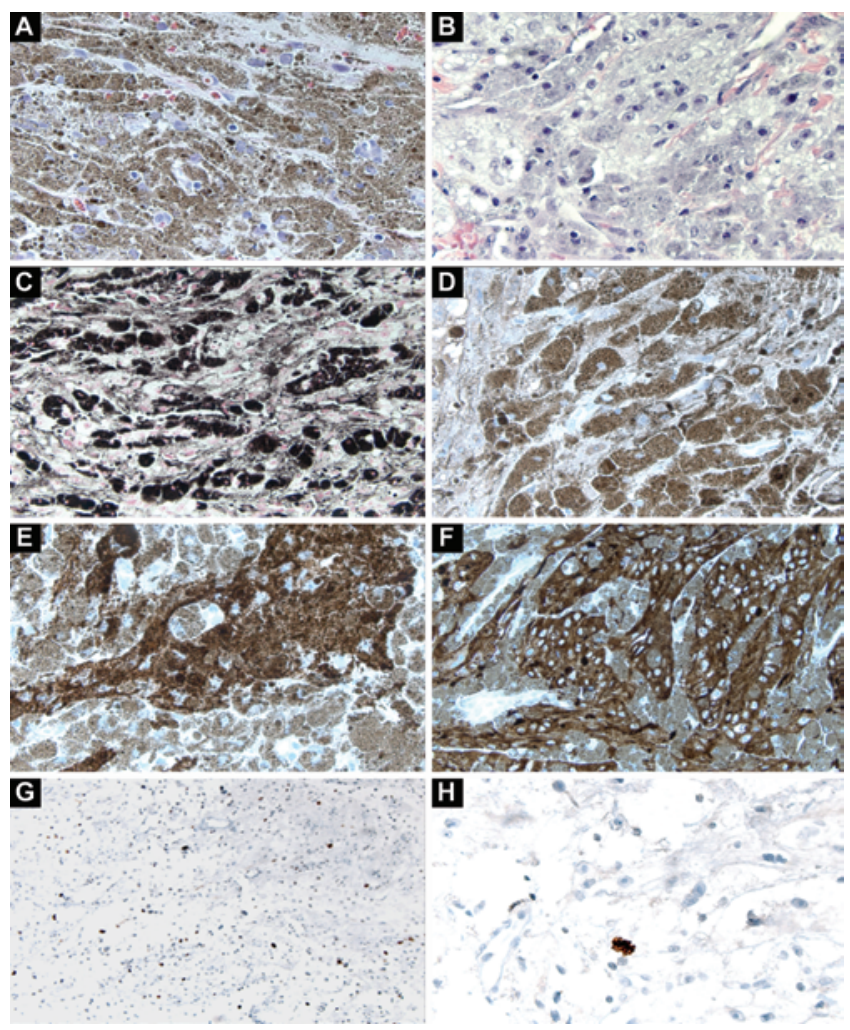

FIG. 2. Photomicrographs of the patient's T-12 neoplasm (primary CNS melanoma). A: Section showing heavily pigmented tumor cells $(\mathrm{H}$ $\& E$, unbleached, original magnification $\times 200$ ). B: Section revealing epithelioid cytomorphology and prominent nucleoli of neoplastic melanocytes ( $H$ \& E, bleached, original magnification original magnification $\times 200$ ). C-H: Masson-Fontana special stain (C, original magnification $\times 200), S 100(D$, original magnification $\times 200), H M B 45(E$, original magnification $\times 200)$, Melan- $A(F$, original magnification $\times 200)$, Ki 67 ( $G$, original magnification $\times 100)$, and phosphohistone $H 3(H$, original magnification $\times 400$ ) highlighting deposits of melanin pigment. Figure is available in color online only.

\section{Second Operation}

The patient was admitted 2 months later for elective resection of the L-5 nerve root lesion via L4-5 laminectomy (Fig. 3). This proceeded without complication, and the resultant pathology was consistent with schwannoma WHO Grade I (Fig. 4A and B). Notably, there was a melanotic appearance of the arachnoid at the level of the L4-5 schwannoma (Fig. 4C and D), with biopsy of the suspicious area showing melanocytosis or benign melanocytoma but no overt melanoma. After an interdisciplinary neurooncology conference review, it was recommended that the patient undergo adjuvant radiotherapy to the residual melanoma resection area at T-12; he declined. He recovered his strength and urinary continence and was without sensory deficits.

\section{Postoperative Course}

Ten months after the initial operation, the patient presented with persistent right leg numbness and "shock-like" sensations with coughing but was functionally intact. Repeat MR imaging demonstrated a remaining right T12L1 lateral recess mass. Serial PET/CT demonstrated a 


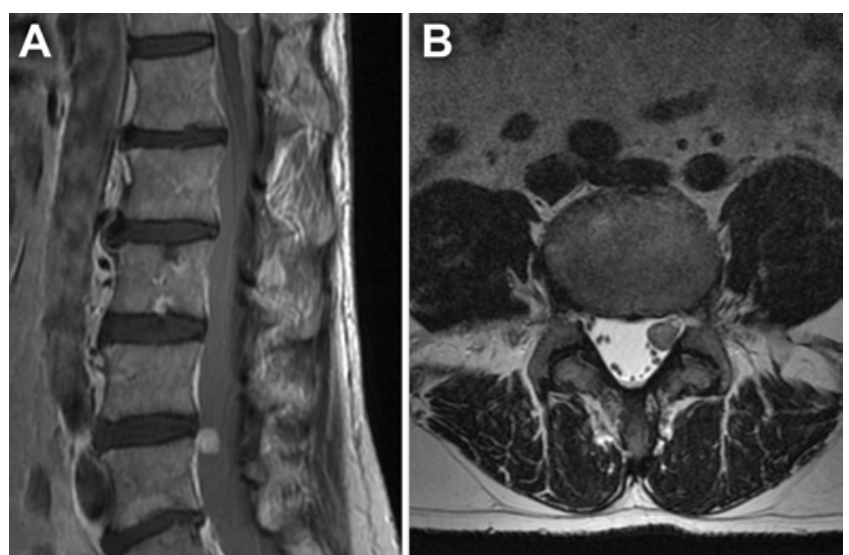

FIG. 3. Orthogonal images of the intradural L-5 lesion. A: T1-weighted postgadolinium parasagittal image showing an avid homogeneous enhancing lesion measuring $0.7 \times 0.9 \times 1 \mathrm{~cm}$. B: Axial T2-weighted image showing the lesion in the lateral recess below the $L-5$ pedicle.

standard uptake value increase of $25 \%$ in the right T-12 pedicle over a period of 4 months, signifying active tumor with no evidence of distant metastatic disease (Fig. 5). Resection of the recurrent melanoma was completed by T-12 costotransversectomy and T10-L3 posterior instrumented fusion. The mass was noted to be completely extradural and was resected en bloc. The patient was again counseled regarding adjuvant radiotherapy and subsequently agreed to undergo therapy. The planning target volume was treated postoperatively with 30 Gy over 5 fractions. At followup 1 year after radiotherapy, the patient was stable with no serious symptoms but occasional back pain. Imaging at that time demonstrated persistent nodular enhancing foci along the dorsal surface of the spinal cord at T-11, T-12, and L-2 with the largest nodule at L-2 measuring $3 \mathrm{~mm}$.

\section{Histopathology and Molecular Examination}

The resection at T-12 demonstrated primary CNS ma-

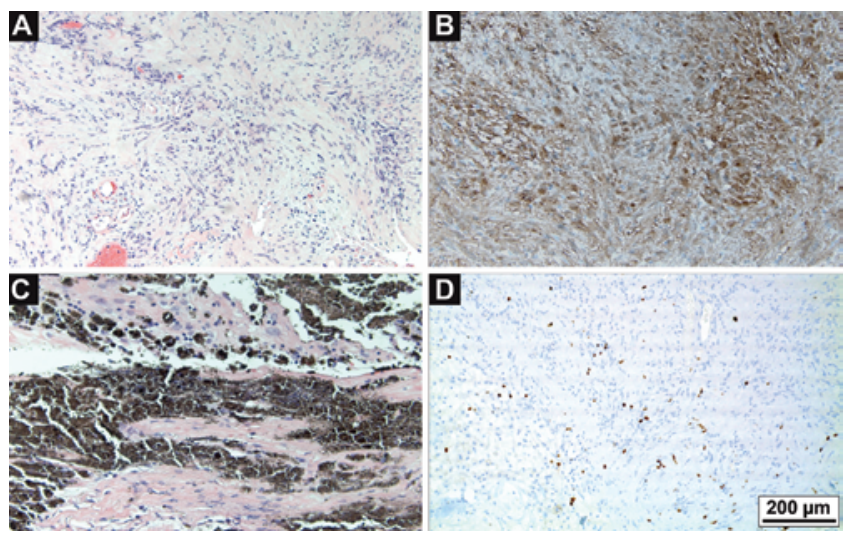

FIG. 4. Photomicrographs of the patient's L4-5 neoplasms (schwannoma and melanocytoma). A: Section showing biphasic histomorphology of conventional schwannoma (H \& E, original magnification $\times 100)$. B: Immunohistochemical stain for S100 demonstrating strong and diffuse positivity in schwannoma cells (original magnification $\times 200$ ). C: Section demonstrating bland population of heavily pigmented tumor cells in arachnoid covering (unbleached, H \& E, original magnification $\times 200$ ). D: Ki 67 proliferative index is very low (approximately 1\%; (unbleached, $H$ \& E, original magnification $\times 100$ ). Figure is available in color online only.
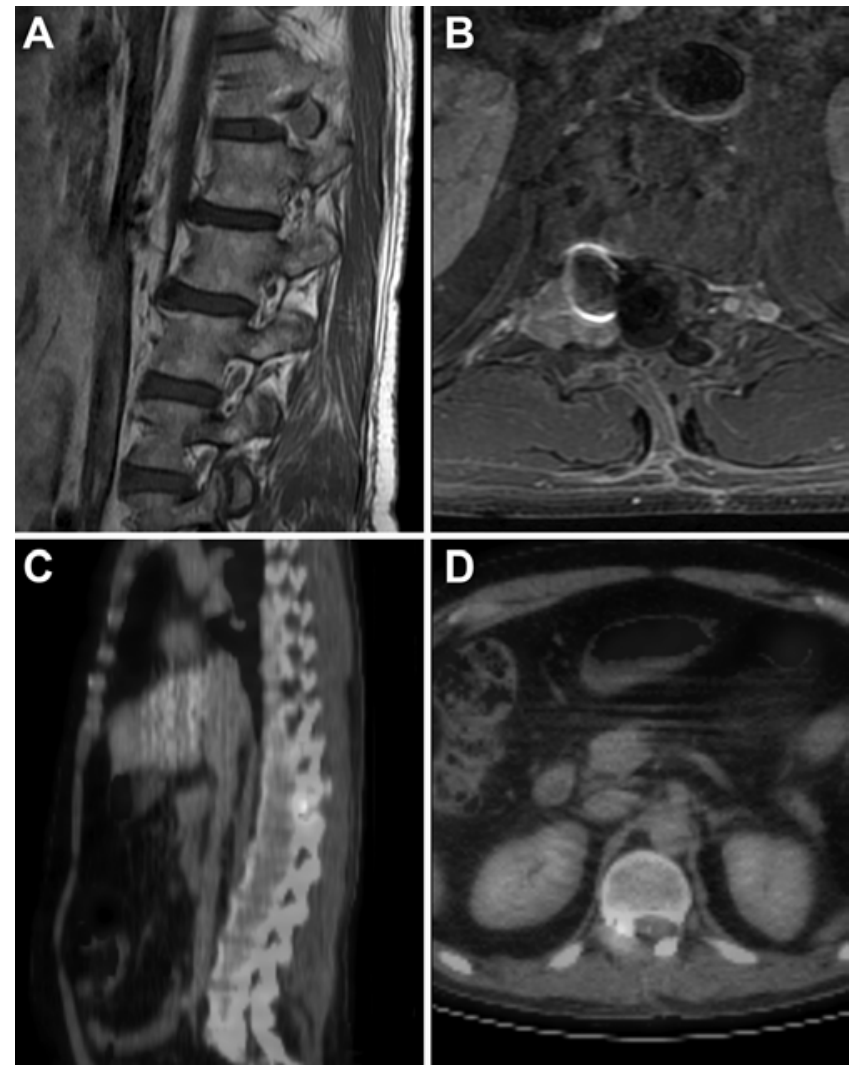

FIG. 5. Orthogonal images from enhanced lumbar MR showing recurrent extraspinal T-12 melanoma. A: Parasagittal T2-weighted/FLAIR image showing recurrent melanoma entirely filling and deforming the T-12 neuroforamina. B: Axial T1-weighted postgadolinium image demonstrating enhancement of the foraminal and extraforaminal lesion along with artifact. C and D: Parasagittal (C) and axial (D) PET/CT images through the T-12 lesion, showing corresponding increased standard uptake value.

lignant melanoma upon histopathological examination. Unbleached H \& E sections revealed heavily pigmented neoplasm composed of solid sheets of epithelioid-looking neoplastic melanocytes with large cherry-red nucleoli and ample cytoplasm that was partly filled with pigment. Masson-Fontana special stain confirmed the identity of the pigment as melanin, and melanocytic markers including S100, HMB-45 and Melan-A were also strongly positive. Epithelial membrane antigen and CAM 5.2 were negative. Mitotic figures were not obvious on routine staining, but phosphohistone H3 immunostaining showed about $5 \mathrm{mi}-$ totic figures per $10 \mathrm{hpf}$. Ki 67 proliferative index was also increased (up to $5 \%-20 \%$ ). The diagnosis of a lower-grade primary CNS melanocytic neoplasm such as melanocytoma or an intermediate-grade melanocytic neoplasm was considered initially, but lack of nesting pattern, prevalence of epithelioid neoplastic melanocytes, prominent nuclear atypia, and increased mitotic rates were supportive of the diagnosis of melanoma. ${ }^{6}$ The specimen obtained from the local recurrence of the tumor showed the same histomorphology. Lack of clinical evidence of melanoma or its metastases elsewhere together with comparatively less significant increase of Ki 67 and quantity of mitotic figures that would be seen in melanoma metastases (17\%-38\% 
and 7-35 mitotic figure/10 hpf, respectively) dismissed the diagnosis of secondary melanoma in our case. ${ }^{3}$

The L-5 intradural tumor demonstrated typical histomorphology of schwannoma, WHO Grade I with hypercellular and hypocellular areas, and strongly positive S100 immunostaining. The biopsied arachnoid covering at the same level showed heavily pigmented melanocytes. The melanocytes were spindle shaped, demonstrated bland histomorphology, and did not show prominent nucleoli. Mitotic figures were absent and Ki 67 proliferative index was minimal $(1 \%)$. This lesion was considered either a discrete melanocytoma or a part of diffuse leptomeningeal melanocytosis because of lack of aggressive features. Neurocutaneous melanosis was ruled out clinically.

The main genes driving oncogenesis in melanomas were evaluated using the bidirectional Sanger sequencing technique for possible targeted therapy use. These genes included BRAF, NRAS, c-KIT, GNAQ, and GNA11. Neither of activating mutations of these genes was found in the primary CNS melanoma and the subsequent recurrence. The second melanocytic lesion at L4-5 was also GNAQ and GNAII negative.

\section{Discussion}

The first reported case of complex neurocristopathy involved 3 tumors of neural crest origin. ${ }^{9}$ A 64 -year-old woman with a medical history of diabetes mellitus and systemic hypertension was referred for evaluation of diabetic retinopathy when a left choroidal juxtapapillary malignant melanoma was discovered in association with a right superolateral periorbital neurofibroma and a right sphenoid wing meningioma. An enucleation was performed for the uveal malignant melanoma, which was revealed to be of mixed cell-type, epithelioid cell-rich juxtapapillary malignant melanoma of the choroid. There was no recurrence up to 1 year postoperatively. Interestingly, the patient had no family history of neurofibromatosis, though the association of neurofibromas and meningiomas has long been recognized in this condition. ${ }^{5}$ Additionally, neurofibromas and meningiomas have both been reported in association with cutaneous malignant melanomas. ${ }^{1,4}$ The report of Warwar et al. was the first to describe both neurofibroma and meningioma with uveal malignant melanoma in the setting of a complex neurocristopathy. ${ }^{9}$

A second report of a complex neurocristopathy involving an ophthalmic melanoma described an 88-year-old woman with no medical history of neurofibromatosis who presented with a choroidal melanoma and ipsilateral optic nerve meningioma. ${ }^{7}$ Choroidal malignant melanoma filling the entire globe with secondary angle closure of the left eye was confirmed via ultrasonography. Circumferential proliferation of meningothelial cells with intranuclear inclusion of the optic nerve sheath demonstrated incidental meningioma. The patient died of metastatic disease 6 months postoperatively.

We report the first patient, to our knowledge, who demonstrated complex neurocristopathy involving a spinal melanoma and schwannoma. A review of the existing literature revealed 2 cases of ophthalmic melanoma in association with other neural crest tumors but no additional cases of spinal melanoma in a complex neurocristopathy context. Primary CNS melanoma is a subset of all primary CNS melanotic tumors and has an estimated incidence less than $1 \%$ of all melanomas, while schwannomas and meningiomas are more commonly treated spinal pathologies. ${ }^{8}$ Upon discovery of a primary CNS malignant melanoma, physicians should be cognizant of other potential tumors of neural crest origin and prepare for the possibility of managing complex neurocristopathy.

\section{Acknowledgments}

We thank Shirley McCartney, $\mathrm{PhD}$, and Andy Rekito, MS, for editorial and illustrative assistance, respectively. This work was supported by the Oregon Health \& Science University Campagna Scholarship to Aaron Chance, BS.

\section{References}

1. Azizi E, Friedman J, Pavlotsky F, Iscovich J, Bornstein A, Shafir R, et al: Familial cutaneous malignant melanoma and tumors of the nervous system. A hereditary cancer syndrome. Cancer 76:1571-1578, 1995

2. Bolande RP: Neurocristopathy: its growth and development in 20 years. Pediatr Pathol Lab Med 17:1-25, 1997

3. Brat DJ, Giannini C, Scheithauer BW, Burger PC: Primary melanocytic neoplasms of the central nervous systems. Am J Surg Pathol 23:745-754, 1999

4. Duve S, Rakoski J: Cutaneous melanoma in a patient with neurofibromatosis: a case report and review of the literature. Br J Dermatol 131:290-294, 1994

5. Eljamel MS, Foy PM: Multiple meningiomas and their relation to neurofibromatosis. Review of the literature and report of seven cases. Surg Neurol 32:131-136, 1989

6. Liubinas SV, Maartens N, Drummond KJ: Primary melanocytic neoplasms of the central nervous system. J Clin Neurosci 17:1227-1232, 2010

7. Mehta P, Agrawal P, Luthert P, Durrani OM: Optic nerve sheath meningioma in a patient with choroidal malignant melanoma: a case report of a complex neurocristopathy. Orbit 28:436-438, 2009

8. Trinh V, Medina-Flores R, Taylor CL, Yonas H, Chohan MO: Primary melanocytic tumors of the central nervous system: Report of two cases and review of literature. Surg Neurol Int 5:147, 2014

9. Warwar RE, Bullock JD, Shields JA, Eagle RC Jr: Coexistence of 3 tumors of neural crest origin: neurofibroma, meningioma, and uveal malignant melanoma. Arch Ophthalmol 116:1241-1243, 1998

\section{Disclosure}

The authors report no conflict of interest concerning the materials or methods used in this study or the findings specified in this paper.

\section{Author Contributions}

Conception and design: Raslan, Chance, Raskin. Acquisition of data: Raslan, Chance, Liu, Raskin. Analysis and interpretation of data: all authors. Drafting the article: all authors. Critically revising the article: Raslan, Liu, Raskin. Reviewed submitted version of manuscript: Raslan, Liu, Raskin, Zherebitskiy, Gultekin. Approved the final version of the manuscript on behalf of all authors: Raslan.

\section{Correspondence}

Ahmed M. Raslan, Department of Neurological Surgery, Oregon Health \& Science University, Mail Code: CH8N, 3303 S.W. Bond Ave., Portland, OR 97239. email: raslana@ohsu.edu. 